\title{
MAPK/p38 regulation of cytoskeleton rearrangement accelerates induction of macrophage activation by TLR4, but not TLR3
}

\author{
HONGJUN BIAN $^{1}$, FEIFEI LI ${ }^{2}$, WENWEN WANG ${ }^{2}$, QI ZHAO $^{2}$, SHANSHAN GAO $^{2}$, JINCAI MA $^{2,3}$, \\ XIAO LI $^{2}$, WANHUA REN ${ }^{2}$, CHENGYONG QIN ${ }^{2}$ and JIANNI QI ${ }^{4}$
}

\begin{abstract}
Departments of ${ }^{1}$ Emergency Medicine and ${ }^{2}$ Gastroenterology, Shandong Provincial Hospital Affiliated to Shandong University, Jinan, Shandong 250021; ${ }^{3}$ Department of Gastroenterology, Jinan Fifth People's Hospital, Jinan, Shandong 250022;

${ }^{4}$ Central Laboratory, Shandong Provincial Hospital Affiliated to Shandong University, Jinan, Shandong 250021, P.R. China
\end{abstract}

Received October 20, 2016; Accepted September 13, 2017

DOI: $10.3892 /$ ijmm.2017.3143

\begin{abstract}
Toll-like receptor 3 (TLR3) and TLR4 utilize adaptor proteins to activate mitogen-activated protein kinase (MAPK), resulting in the acute but transient inflammatory response aimed at the clearance of pathogens. In the present study, it was demonstrated that macrophage activation by lipopolysaccharide (LPS) or poly(I:C), leading to changes in cell morphology, differed significantly between the mouse macrophage cell line RAW264.7 and mouse primary peritoneal macrophages. Moreover, the expression of $\alpha$ - and $\beta$-tubulin was markedly decreased following LPS stimulation. By contrast, $\alpha$ - and $\beta$-tubulin expression were only mildly increased following poly(I:C) treatment. However, the expression of $\beta$-actin and GAPDH was not significantly affected. Furthermore, it was verified that vincristine pretreatment abrogated the cytoskeleton rearrangement and decreased the synthesis and secretion of proinflammatory cytokines and migration of macrophages caused by LPS. Finally, it was observed that the MAPK/p38 signaling pathway regulating cytoskeleton rearrangement may participate in LPS-induced macrophage cytokine production and migration. Overall, the findings of the present study indicated that MAPK/p38 regulation of the cytoskeleton, particularly tubulin proteins, plays an important role in LPS-induced inflammatory responses via alleviating the synthesis and secretion of proinflammatory cytokines and inhibiting the migration of macrophages.
\end{abstract}

\section{Introduction}

Innate immune signaling receptors play a pivotal role in defense against invasion by pathogenic microorganisms or tissue damage (1). Toll-like receptors (TLRs), as one of

Correspondence to: Dr Jianni Qi, Central Laboratory, Shandong Provincial Hospital Affiliated to Shandong University, 324 Jingwu Road, Jinan, Shandong 250021, P.R. China

E-mail: slqijn@126.com

Key words: Toll-like receptor 3/4, cytoskeleton, macrophage activation, vincristine, mitogen-activated protein kinase the important innate immune signaling receptors, initiate complicated signaling pathways leading to acute but transient inflammatory responses aimed at the clearance of pathogens and cellular debris (2). TLR3 and TLR4 recruit TIR-domain-containing adapter-inducing interferon- $\beta$ (TRIF) and/or MyD88, leading to the expression of proinflammatory cytokines and the induction of interferon (IFN)- $\beta(2,3)$. Although proinflammatory cytokines and IFN- $\beta$ are essential for resistance against invading pathogens, uncontrolled TLR3/4 activation by poly(I:C)/lipopolysaccharide (LPS) and production of proinflammatory cytokines, such as tumor necrosis factor (TNF)- $\alpha$, interleukin (IL)- 6 and IFN- $\beta$ are the main cause of septic shock (4). Therefore, elucidating the mechanisms underlying the production of these cytokines is crucial for developing treatments for such conditions.

The cytoskeleton plays an important role in modulating cell morphology, migration and division through contractile ring formation and certain intracellular signaling pathways (5). Recent studies have provided evidence supporting the importance of the cytoskeleton in immunocytes, including macrophage-, B-cell- and dendritic cell-mediated inflammation (6-8). poly(I:C) and LPS are two TLR3/4 agonists commonly used to induce macrophage activation and investigate the effect of other signaling molecules in cellular responses (3). However, the role of the cytoskeleton in the TLR3/4 signaling pathway inducing macrophage activation has not been clearly determined.

Vincristine (VCR) is a vinca alkaloid extracted from the plant Catharanthus roseus (9), which has long been used as a chemotherapeutic agent for the treatment of childhood and adult acute lymphocytic leukemia, Hodgkin's and non-Hodgkin's lymphoma, and various solid tumors, including germ cell tumors, small-cell lung cancer, Ewing's sarcoma, neuroblastoma, breast cancer, melanoma and multiple myeloma $(10,11)$. VCR may lead to microtubule depolymerization via binding to the tubulin protein in a dose-dependent manner, similar to colchicine (12). However, the effect of VCR on macrophage activation by poly(I:C) and LPS has not been fully elucidated.

The aim of the present study was to demonstrate that macrophage activation, leading to cytokine production and changes in cell morphology, differs significantly between LPS and poly(I:C). In addition, tubulin expression was decreased 
following LPS stimulation. By contrast, tubulin expression was somewhat increased following poly(I:C) treatment. There was no significant effect on the expression of $\beta$-actin and glyceraldehyde 3-phosphate dehydrogenase (GAPDH) in LPS- or poly(I:C)-induced macrophages. Furthermore, it was observed that VCR pretreatment represses the cytoskeleton rearrangement in macrophages and reduces the production of proinflammatory cytokines and cell migration caused by LPS. In addition, it was demonstrated that the mitogen-activated protein kinase (MAPK)/p38 signaling pathway plays a pivotal role in cytoskeleton rearrangement leading to cytokine production and macrophage invasion induced by LPS.

\section{Materials and methods}

Reagents and preparation. LPS (Escherichia coli, 055:B5) was obtained from Sigma-Aldrich (Merck KGaA, St. Louis, MO, USA; cat. no. L2880). poly(I:C) was obtained from InvivoGen (San Diego, CA, USA; cat. no. tlrl-picw). VCR was purchased from Dalian Meilun Biotech Co.,Ltd. (Dalian, Liaoning, China; cat. no. 2068-78-2). Mouse monoclonal antibodies to GAPDH (cat. no. 60004-1-lg), $\beta$-actin (cat. no. 60008-1-lg), $\alpha$-tubulin (cat. no. 66031-1-lg) and $\beta$-tubulin (cat. no. 66240-1-lg) were purchased from Proteintech Group, Inc. (Rosemont, IL, USA). LPS and poly(I:C) were dissolved and diluted with corresponding liquid according to the manufacturer's instructions. The VCR was stored as powder at room temperature. For cytology experiments, VCR was dissolved in a stock solution of sterile deionized $\mathrm{H}_{2} \mathrm{O}$ to a concentration of $1 \mathrm{mg} / \mathrm{ml}$. For further experiments, stock solution was diluted in sterile deionized with $\mathrm{H}_{2} \mathrm{O}$ or Dulbecco's modified Eagle's medium (DMEM; Gibco-BRL; Thermo Fisher Scientific, Grand Island, NY, USA) containing $10 \%$ fetal bovine serum (FBS; Gibco ${ }^{\circledR}$ Sera; Thermo Fisher Scientific, Newcastle, Australia) to a final concentration working solution of 10, 20, 50, 100,300, 500 and $1,000 \mathrm{ng} / \mu 1$.

Mice and cells. Male C57BL/6J mice, 6-8 weeks old, were obtained from the Animal Research Committee of the Institute of Biology and Cell Biology (Shanghai, China) and housed in a specific pathogen-free environment. The animal room was kept at $20-22^{\circ} \mathrm{C}$ under a $12-\mathrm{h}$ light/dark cycle. All animal experiments were conducted in accordance with the National Institutes of Health Guide for the Care and Use of Laboratory Animals, with the approval of the Scientific Investigation Board of the Medical School of Shandong University (Jinan, China). Mouse primary peritoneal macrophages were prepared as previously described (3). The mouse macrophage cell line RAW264.7 was obtained from American Type Culture Collection (Manassas, VA, USA) and cultured in DMEM containing $10 \%(\mathrm{v} / \mathrm{v}) \mathrm{FBS}, 100 \mathrm{U} / \mathrm{ml}$ penicillin and $100 \mu \mathrm{g} /$ ml streptomycin (Gibco-BRL; Thermo Fisher Scientific, Grand Island, NY, USA). The cell lines were maintained at $37^{\circ} \mathrm{C}$ in a humidified incubator with $5 \% \mathrm{CO}_{2}$. RAW264.7 cells were stimulated with $100 \mathrm{ng} / \mathrm{ml}$ LPS or $20 \mu \mathrm{g} / \mathrm{ml}$ poly(I:C) for different times, with or without pretreatment with VCR or MAPK pathway inhibitors.

RNA extraction and quantification. Total RNA was extracted using TRIzol reagent (Invitrogen; Thermo Fisher Scientific,
Carlsbad, CA, USA) according to the manufacturer's instructions, and reverse transcription was performed using a Takara reverse transcription kit (Takara, Shiga, Japan). The expression of IL-6, TNF- $\alpha$ and IFN- $\beta$ was quantified using SYBR Premix Ex Tap ${ }^{\mathrm{TM}}$, with GAPDH as an internal normalized reference. The specific sequences of the primers used were as previously described $(3,13)$. Quantitative polymerase chain reaction (qPCR) was performed under the following conditions: $95^{\circ} \mathrm{C}$ for $30 \mathrm{sec}$, followed by 45 cycles at $95^{\circ} \mathrm{C}$ for $5 \mathrm{sec}, 60^{\circ} \mathrm{C}$ for $5 \mathrm{sec}, 72^{\circ} \mathrm{C}$ for $5 \mathrm{sec}$ and $65^{\circ} \mathrm{C}$ for $20 \mathrm{sec}$, using the LightCycler Real-time PCR system (Roche Diagnostics, Indianapolis, IN, USA) as previously described (14).

Enzyme-linked immunosorbent assay (ELISA). The cell culture supernatants were collected, and the concentration of IL-6 (cat. no. KMC0061) and TNF- $\alpha$ (cat. no. KMC3011) were measured using a commercially available ELISA kit (all from Invitrogen; Thermo Fisher Scientific), in accordance with the manufacturer's instructions.

Boyden chamber assays. The Boyden chamber was obtained from Corning, Inc. (Corning, NY, USA; cat. no. 3422). DMEM $(600 \mu \mathrm{l})$ with $10 \% \mathrm{FBS}$ was added to the lower chamber. Next, RAW264.7 cells were collected and washed with DMEM. The cells were resuspended in $200 \mu \mathrm{l}$ of DMEM with/without VCR and/or LPS, and then seeded to the upper chamber at a concentration of $2 \times 10^{5}$ cells/well. Following incubation for $20 \mathrm{~h}$ at $37^{\circ} \mathrm{C}$ in a humidified incubator with $5 \% \mathrm{CO}_{2}$, the Boyden chamber was fixed with $4 \%$ paraformaldehyde. Non-migrated cells on the upper surface of the filter were removed with a cotton swab and the cells that traversed and spread on the lower surface of the membrane were stained with hematoxylin. The filter was dehydrated with gradient alcohol and dried at room temperature. These membranes were sealed using neutral gum and cell invasiveness was observed under a light microscope as previously described (14).

Cell counting kit-8 (CCK-8) assay. Cell proliferation was evaluated using CCK-8 (Dojindo Molecular Technologies, Inc., Kumamoto, Japan). Cells were seeded at a density of $3 \times 10^{4} /$ well in 96 -well plates and were then treated with VCR at different concentrations. After 0, 2, 4, 8, 12, 24, 36 and $48 \mathrm{~h}$, $20 \mu \mathrm{l}$ of CCK-8 solution was added to each well and incubated at $37^{\circ} \mathrm{C}$ for $1 \mathrm{~h}$. At the end of the incubation, the optical density was read at $450 \mathrm{~nm}$ using a microplate reader (Thermo Fisher Scientific, Waltham, MA, USA). The mean values were determined from different wells as previously described (14).

Confocal microscopy analysis. RAW264.7 cells were grown on coverslips and stimulated with $100 \mathrm{ng} / \mathrm{ml}$ LPS or $20 \mu \mathrm{g} / \mathrm{ml} \operatorname{poly}(\mathrm{I}: \mathrm{C})$ for $24 \mathrm{~h}$ at $37^{\circ} \mathrm{C}$, with or without VCR pretreatment. Non-adherent cells were then washed away with phosphate-buffered saline (PBS). The cells were fixed in $4 \%$ paraformaldehyde for $15 \mathrm{~min}$ and permeabilized with $0.1 \%$ Triton $\mathrm{X}-100$. After blocking with $10 \%$ bovine serum albumin (BSA) in PBS for $2 \mathrm{~h}$ at room temperature, they were incubated with primary antibodies against $\alpha$-tubulin, $\beta$-tubulin and phalloidin-FITC (50 $\mu \mathrm{g} / \mathrm{ml}$; cat. no. P5282; Sigma Aldrich; Merck $\mathrm{KGaA}$ ) for $1 \mathrm{~h}$ at room temperature, followed by washing 3 times with PBS for $5 \mathrm{~min} / \mathrm{time}$. Cells were incubated with 
secondary antibodies (cat. no. CA11005s; Invitrogen; Thermo Fisher Scientific) for $1 \mathrm{~h}$ at room temperature in the dark, except for the phalloidin-FITC staining groups, followed by washing 3 times with PBS for $5 \mathrm{~min} /$ time. Cell nuclei were stained with DIPA for $8 \mathrm{~min}$ and washed 3 times with PBS for $5 \mathrm{~min} /$ time. After sealing the coverslips with neutral gum, images were captured using a high sensitivity laser scanning confocal microscope (LSM780; Zeiss, Jena, Germany) with the appropriate filters and laser $(488,561$ and $633 \mathrm{~nm})$ and a x63 objective lens.

Simple western assays. Cells were lysed with the CelLytic ${ }^{\mathrm{TM}}$ Cell Lysis Reagent (Sigma Aldrich; Merck KGaA) supplemented with a protease inhibitor cocktail; the protein concentrations were measured on a Multiskan GO microplate reader (Thermo Fisher Scientific) using the bicinchoninic acid assay and BSA standards (Pierce, Rockford, IL, USA), and the volumes were then made equal using the extraction reagent. Automated capillary western blot analyses were performed according to the ProteinSimple user manual provided by the manufacturer. In brief, cell lysate samples were diluted with $1 \mathrm{X}$ sample buffer to $0.2 \mathrm{mg} / \mathrm{ml}$; the diluted samples were then mixed at 1:4 ratio with $5 \mathrm{X}$ master mix (ProteinSimple) containing $1 \mathrm{X}$ fluorescent molecular weight markers, sodium dodecyl sulfate and dithiothreitol, and were then incubated at $98^{\circ} \mathrm{C}$ for $10 \mathrm{~min}$ for protein denaturation. The biotinylated ladder, treated samples, blocking buffer, primary antibodies, horseradish peroxidase-conjugated secondary antibodies, luminol-peroxidase 1:1 mix and wash buffer were dispensed to designated assay plate with 13-Capillary Cartridge (PS-CC02). Chemiluminescence was detected at 5, 15, 30, 60, 120,240 and $480 \mathrm{sec}$. All the primary antibodies were used at 1:50 dilution for simple western blot analysis.

Statistical analysis. All data are presented as the result of three or four independent experiments. All data are expressed as mean \pm standard deviation, and analyzed via one-way analysis of variance and two-tailed Student's t-test using SPSS 16.0 statistical software (SPSS, Inc., Chicago, IL, USA). In all cases, P-values $<0.05$ were considered to indicate statistically significant differences.

\section{Results}

LPS stimulation, but not poly(I:C) stimulation, leads to changes in macrophage morphology. In order to investigate the possibly different mechanism of TLR3/4-induced macrophage activation, the mouse macrophage cell line RAW264.7 and mouse primary peritoneal macrophages were stimulated with LPS or poly(I:C) at different timepoints. First, the activating effect of LPS or poly(I:C) was examined by PCR using IL- 6 and IFN- $\beta$, well-known downstream molecules in the signaling pathway of TLR3/4. As shown in Fig. $1 \mathrm{~A}$ and $\mathrm{B}$, the synthesis of IL- 6 and IFN- $\beta$ was markedly increased with LPS or poly(I:C) stimulation. The expression of other cytokines, such as TNF- $\alpha$ and inducible nitric oxide synthase, was also upregulated (Fig. 1A). However, surprisingly, the expression of the abovementioned cytokines induced by poly(I:C) stimulation was always lower compared with that of the LPS group at any given timepoint. Moreover, TLR3 and TLR4 expression was detected with the PCR assay, and TLR3 and TLR4 were found to be expressed in RAW264.7 cells and mouse primary peritoneal macrophages, although the level of TLR3 was lower compared with that of TLR4 (Fig. 1C). In addition, there was a major distinction between the two groups regarding cell morphology. As shown in Fig. 1D, the cells stimulated by LPS were more stretched and developing multiple pseudopodia or flattened. In sharp contrast, poly(I:C)-induced cells maintained their round morphology, without marked changes compared with the unstimulated group. Subsequently, cytoskeleton rearrangement following LPS or poly(I:C) treatment was observed using a confocal microscope. As shown in Fig. 1E, the cytoskeleton was found to be markedly rearranged via staining of the $\alpha$ - and $\beta$-tubulin and F-actin. Finally, the expression of cytoskeletal proteins ( $\alpha$ - and $\beta$-tubulin and $\beta$-actin) and non-cytoskeletal proteins (GAPDH) was evaluated following LPS or poly(I:C) stimulation using simple western assays. Surprisingly, LPS stimulation significantly reduced $\alpha$ - and $\beta$-tubulin expression, but not that of $\beta$-actin and GAPDH. By contrast, $\alpha$ - and $\beta$-tubulin expression were mildly increased, whereas there was no significant effect on the expression of $\beta$-actin and GAPDH following poly(I:C) stimulation (Fig. 1F). Taken together, these data indicate that cytokine and cytoskeletal protein expression and cell morphology differed significantly following TLR3/4-induced macrophage activation.

$V C R$ pretreatment represses the cytoskeleton rearrangement induced by LPS. VCR may combine with tubulin, actin and 10-nm filament proteins to inhibit cytoskeletal rearrangement, and plays an important role in the cytoskeleton and signaling pathways, such as colchicine. Therefore, in order to further confirm the effect of cell morphological changes on macrophage activation induced by LPS, the mouse macrophage cell line RAW264.7 was pretreated with gradually increasing concentrations of VCR for $30 \mathrm{~min}$, and then stimulated with $100 \mathrm{ng} / \mathrm{ml}$ LPS for 12 or $24 \mathrm{~h}$. As shown in Fig. 2A, a concentration as low as $10 \mathrm{ng} / \mathrm{ml}$ was able to significantly inhibit LPS-induced cell morphological changes and the cells maintained a round shape, rather than developing cytoplasmic projections. Furthermore, cytoskeleton rearrangement following VCR pretreatment was observed using a confocal microscope. As shown in Fig. 2B, the arrangement of $\alpha$ - and $\beta$-tubulin and F-actin was found to be disordered and the cytoskeleton rearrangement was significantly inhibited following VCR pretreatment and LPS stimulation for $24 \mathrm{~h}$. Taken together, these data indicate that VCR pretreatment repressed the cytoskeleton rearrangement induced by LPS.

VCR pretreatment reduces the synthesis and secretion of cytokines and attenuates the migration of macrophages. To investigate the status of macrophage activation during the inhibition of cell morphological changes, the expression and secretion of TNF- $\alpha$ and IL- 6 was detected with PCR and ELISA. As shown in Fig. 3A and B, in RAW264.7 cells, VCR priming markedly decreased the expression and secretion of IL-6 in a dose-dependent manner. However, VCR priming diminished the secretion of TNF- $\alpha$, but not its expression. As shown in Fig. 3C and D, the expression and secretion of IL-6 and TNF- $\alpha$ were all decreased in mouse primary peritoneal 

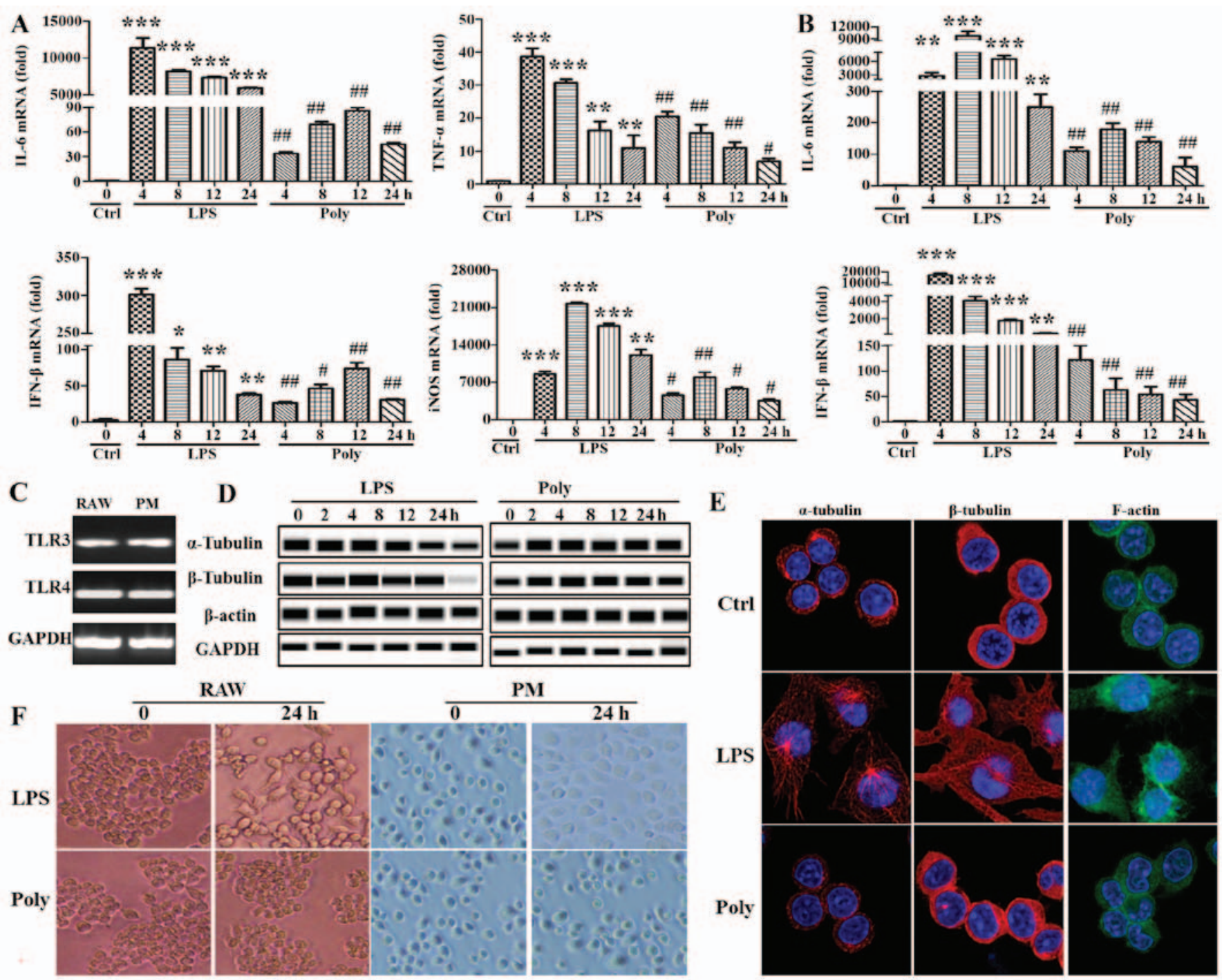

Figure 1. Lipopolysaccharide (LPS) stimulation, but not poly(I:C) stimulation, led to changes in macrophage morphology. (A) RAW264.7 cells and (B) mouse primary peritoneal macrophages (PM) were stimulated with LPS (100 ng/ml) or poly(I:C) $(20 \mu \mathrm{g} / \mathrm{ml})$ for $0,4,8,12$ and $24 \mathrm{~h}$, and interleukin (IL)-6, interferon (IFN)- $\beta$, tumor necrosis factor (TNF)- $\alpha$ and inducible nitric oxide synthase (iNOS) expression was detected with quantitative polymerase chain reaction (PCR). ${ }^{*} \mathrm{P}<0.05,{ }^{* *} \mathrm{P}<0.01,{ }^{* * * *} \mathrm{P}<0.001,{ }^{\#} \mathrm{P}<0.05$ and $^{\# \#} \mathrm{P}<0.01$. (C) Toll-like receptor (TLR)3 and TLR4 expression was detected with the PCR assay in RAW264.7 cells and mouse PM. (D) The expression of $\alpha$ - and $\beta$-tubulin and $\beta$-actin and glyceraldehyde 3-phosphate dehydrogenase (GAPDH) were measured at indicated timepoints with simple western assays. Similar observations were obtained from three independent experiments. (E) After RAW264.7 cells were stimulated with LPS (100 ng/ml) or poly(I:C) $(20 \mu \mathrm{g} / \mathrm{ml})$ for $24 \mathrm{~h}, \alpha$ - and $\beta$-tubulin and F-actin were stained and detected using a confocal microscope. (F) Cell morphology was observed under a light microscope after LPS (100 ng/ml) or poly(I:C) (20 $\mu \mathrm{g} / \mathrm{ml})$ treatment for $24 \mathrm{~h}$ (original magnification, x100).

macrophages during VCR pretreatment. Macrophage invasion is a key factor in the inflammatory response; hence, their invasion ability was detected with Boyden chamber assays. As shown in Fig. 3E, macrophage invasion was significantly accelerated following LPS stimulation. However, only few cells invaded through the filter membrane in the poly(I:C) group. VCR pretreatment remarkably abrogated macrophage invasion caused by LPS, but not poly(I:C). Taken together, these data indicate that VCR pretreatment inhibited the LPS-induced cytokine expression and secretion and attenuated invasion in macrophages.

VCR treatment at low concentrations and for a short time does not inhibit cell proliferation. In order to verify that the inhibition of proinflammatory cytokine expression and secretion and cell invasion in RAW264.7 macrophages by VCR pretreatment was not due to VCR decreasing cell proliferation, the macrophages were pretreated with increasing concentrations of $\operatorname{VCR}(0,10,20,50,100,300,500$ and 1,000 ng/ml). As shown in Fig. 4A, high or low concentrations of VCR did not significantly affect cell proliferation within $12 \mathrm{~h}$. However, over time, VCR at $\geq 100 \mathrm{ng} / \mathrm{ml}$ achieved a higher cell mortality rate. Following VCR primed for $36 \mathrm{~h}$, low concentrations of VCR were also cytotoxic to a certain extent. As shown in Fig. 4B, VCR at $<50 \mathrm{ng} / \mathrm{ml}$ did not significantly affect the cell count for $24 \mathrm{~h}$. However, the cytotoxic effect was markedly increased at VCR concentrations of $\geq 100 \mathrm{ng} / \mathrm{ml}$. Taken together, these data indicate that VCR pretreatment at low doses and for a short time did not significantly affect cell proliferation and activity.

The MAPK/p38 signaling pathway plays a predominant role in cytoskeleton rearrangement by augmenting cytokine produc- 
A

$\mathbf{0 ~ h ~}$

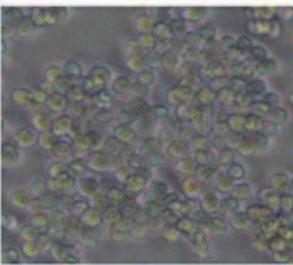

$12 \mathrm{~h}$

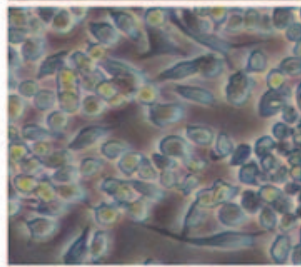

$24 \mathrm{~h}$

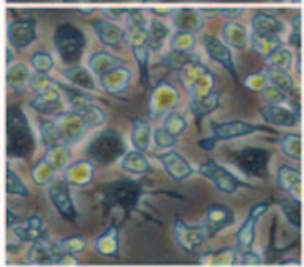

10

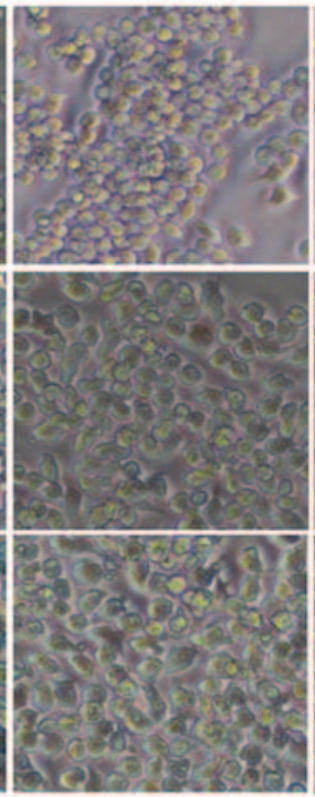

20

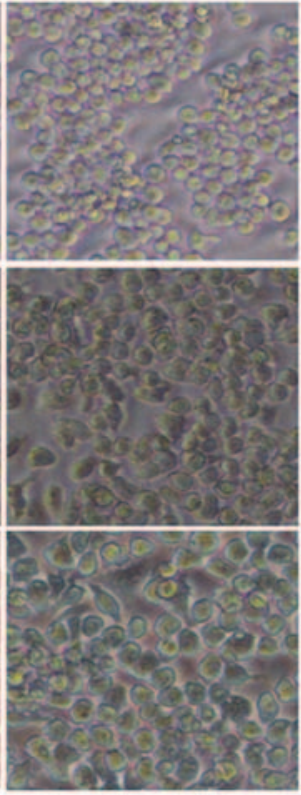

$50(\mathrm{ng} / \mathrm{ml})$

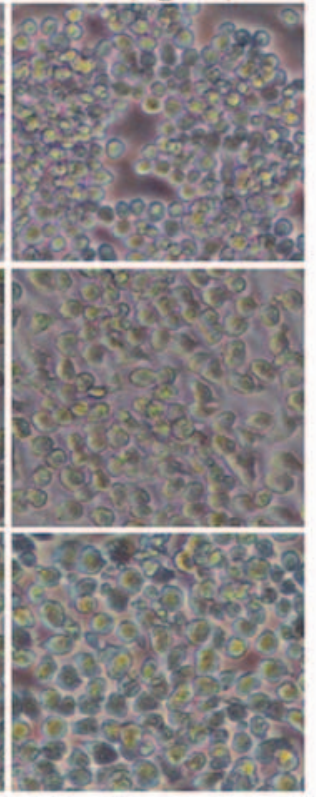

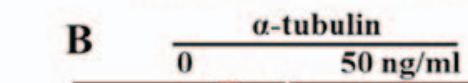
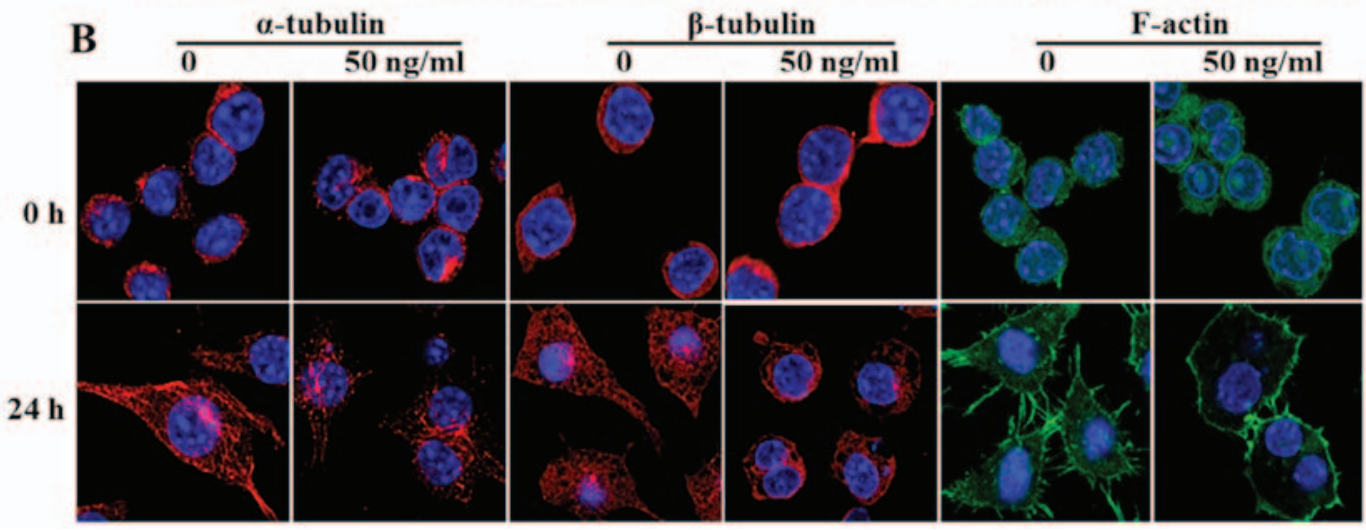

Figure 2. Vincristine (VCR) inhibits cell morphological changes induced by lipopolysaccharide (LPS). (A) After RAW264.7 cells were primed for 30 min with VCR at different concentrations, followed by treatment for 12 and $24 \mathrm{~h}$ with LPS (100 ng/ml), cell morphology was observed under a light microscope (original magnification, x100). (B) After RAW264.7 cells were primed for $30 \mathrm{~min}$ with VCR at $50 \mathrm{ng} / \mathrm{ml}$ followed by treatment for $24 \mathrm{~h}$ with LPS (100 ng/ml), $\alpha$ - and $\beta$-tubulin and F-actin were stained and detected using a confocal microscope. Similar observations were obtained from three independent experiments.

tion induced by LPS. TLR4 initiates the MyD88-dependent and -independent pathway that involves TRAF6 to activate the MAPK signaling pathway, resulting in phosphorylation of the extracellular signal-regulated kinase (ERK), p38 and c-Jun N-terminal kinase (JNK), with subsequent nuclear translocation to regulate proinflammatory cytokine transcription (15). PD98059, SB203580 and SP600125 are effective inhibitors downregulating the phosphorylation level of MAPK signaling pathway molecules (16). To determine the role of the MAPK signaling pathway in cytoskeleton rearrangement caused by LPS, the macrophages were respectively pretreated with these inhibitors for $30 \mathrm{~min}$, and then stimulated with $100 \mathrm{ng} / \mathrm{ml} \mathrm{LPS}$. As shown in Fig. 5A, ERK, p38 and JNK phosphorylation were markedly increased following LPS stimulation, which was consistent with previously reported data on LPS inducing MAPK activation (16). Treatment with the abovementioned inhibitors significantly attenuated LPS-induced ERK, p38 and JNK phosphorylation. Next, the effect of these inhibitors on LPS-induced cytokine expression was further investigated. As shown in Fig. 5B, LPS increased the levels of IL-6 and IFN- $\beta$ mRNA following stimulation for $4 \mathrm{~h}$. This increase was reversed by treatment with SB203580 and SP600125, but not PD98059. Furthermore, the effect of these inhibitors on macrophage morphology was assessed. As shown in Fig. 5C, LPS induced cell stretching after stimulation for 12 and $24 \mathrm{~h}$. This effect was abrogated by treatment with SB203580, but not PD98059 and SP600125. Moreover, the phosphorylation level of p38 and p65 was measured after VCR pretreatment followed by LPS stimulation. As shown in Fig. 5D, p38 phosphorylation was lower following VCR pretreatment; however, there was little change in the phosphorylation of p65. Taken together, these data indicate that the MAPK/p38 signaling pathway may play a prominent role in cytoskeleton rearrangement leading to cytokine production induced by LPS.

\section{Discussion}

The present study demonstrated a striking difference in cytokine production and cell morphology between LPS- and poly(I:C)-induced macrophage activation. Stimulation by LPS, but not poly(I:C), significantly decreased tubulin protein expression, leading to cytoskeleton rearrangement, while the 

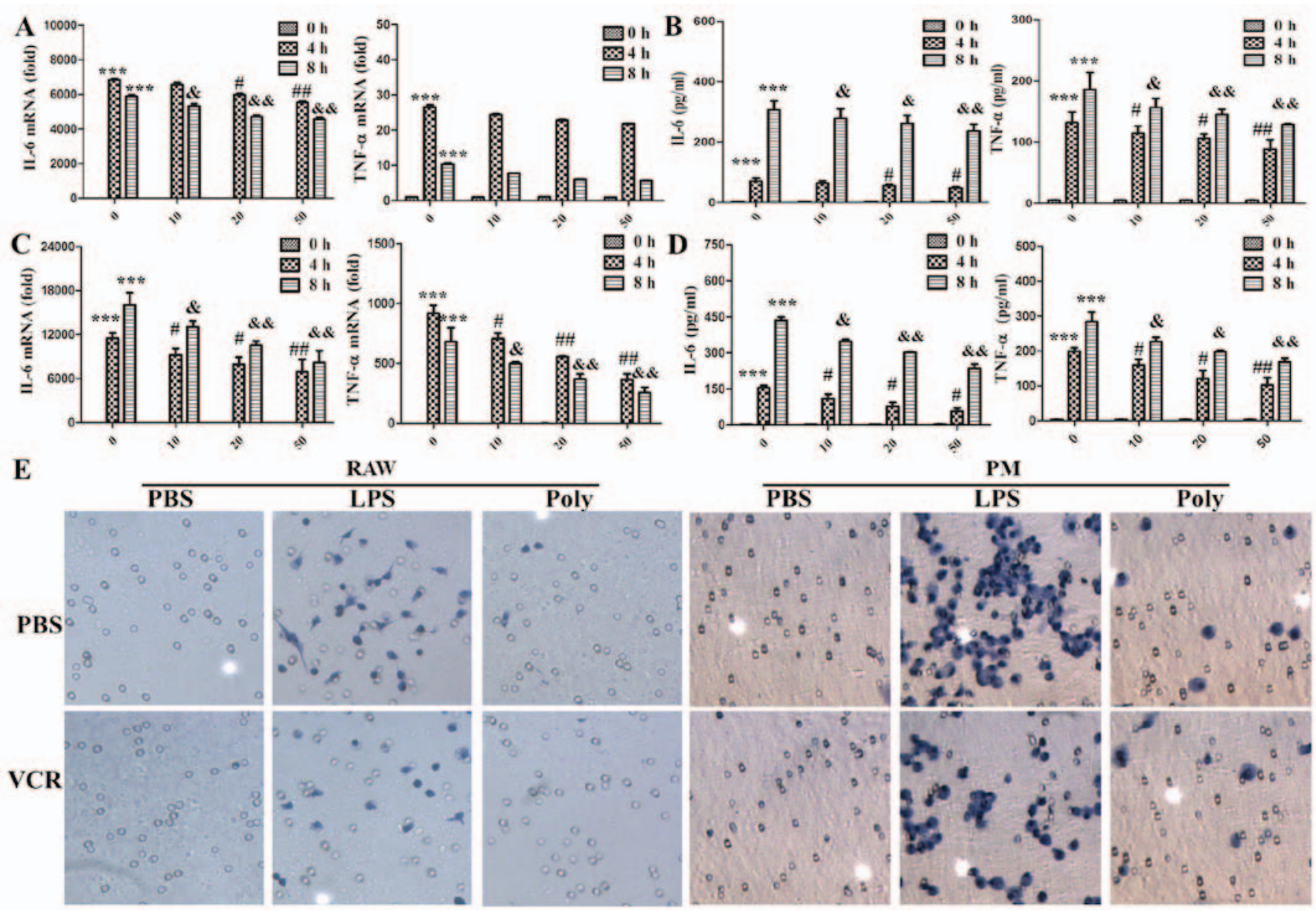

Figure 3. Vincristine (VCR) pretreatment reduced the synthesis and secretion of cytokines and cell migration caused by lipopolysaccharide (LPS). (A and B) RAW264.7 cells were primed for $30 \mathrm{~min}$ with VCR at different concentrations and then treated for 0,4 and $8 \mathrm{~h}$ with $100 \mathrm{ng} / \mathrm{ml} \mathrm{LPS}$. (A) Interleukin (IL)-6 and tumor necrosis factor (TNF)- $\alpha$ expression was measured by quantitative polymerase chain reaction (PCR). ${ }^{* * *} \mathrm{P}<0.001,{ }^{\#} \mathrm{P}<0.05$, ${ }^{\#} \mathrm{P}<0.01,{ }^{\&} \mathrm{P}<0.05$ and ${ }^{\&}{ }^{\&} \mathrm{P}<0.01$. (B) Secretion of IL-6 and TNF- $\alpha$ in the cell culture supernatants was determined by ELISA $\left({ }^{* * * *} \mathrm{P}<0.001,{ }^{*} \mathrm{P}<0.05\right.$, ${ }^{\text {,\# }} \mathrm{P}<0.01,{ }^{\text {\& }} \mathrm{P}<0.05$ and $\left.{ }^{\& \&} \mathrm{P}<0.01\right)$. (C and D) Peritoneal macrophages were primed for $30 \mathrm{~min}$ with VCR at different concentrations and then treated for $0,4 \mathrm{and} 8 \mathrm{~h}$ with $100 \mathrm{ng} / \mathrm{ml} \mathrm{LPS}$. (C) IL-6 and TNF- $\alpha$ expression was measured by quantitative PCR $\left({ }^{* * *} \mathrm{P}<0.001,{ }^{*} \mathrm{P}<0.05,{ }^{\# \#} \mathrm{P}<0.01,{ }^{\&} \mathrm{P}<0.05\right.$ and $\left.{ }^{\& \&} \mathrm{P}<0.01\right)$. (D) Secretion of IL-6 and TNF- $\alpha$ in the cell culture supernatants was determined by ELISA $\left({ }^{* * *} \mathrm{P}<0.001,{ }^{\sharp} \mathrm{P}<0.05,{ }^{\# \#} \mathrm{P}<0.01,{ }^{\&} \mathrm{P}<0.05\right.$ and $\left.{ }^{\& \&} \mathrm{P}<0.01\right)$. The data are shown as mean \pm standard deviation of triplicate samples. (E) After RAW264.7 cells and peritoneal macrophages (PM) were primed for $30 \mathrm{~min}$ with $50 \mathrm{ng} / \mathrm{ml} \mathrm{VCR}$ and then treated for $20 \mathrm{~h}$ (RAW264.7) or $36 \mathrm{~h}(\mathrm{PM})$ with $100 \mathrm{ng} / \mathrm{ml}$ LPS and poly(I:C) $(20 \mu \mathrm{g} / \mathrm{ml})$, migrated cells on the lower surface of the membrane were stained with hematoxylin (original magnification, x200). Similar observations were obtained from three independent experiments. PBS, phosphate-buffered saline.

expression of actin and GAPDH were not significantly affected. VCR, a drug accelerating microtubule depolymerization via tubulin binding, was found to inhibit cytoskeleton rearrangement in LPS-induced macrophages, decrease the production of proinflammatory cytokines and diminish macrophage migration. Furthermore, the MAPK/p38 signaling pathway may play a prominent role in cytoskeleton rearrangement leading to cytokine production induced by LPS. Our data suggest that the cytoskeleton regulates LPS-induced macrophage activation via the MAPK/p38 signaling pathway.

Macrophages represent a heterogeneous population of immune cells with various functions in body homeostasis and disease initiation, maintenance and resolution $(17,18)$. Macrophages express a myriad of pattern recognition receptors (PRRs), including TLRs, that enable them to rapidly respond to pathogen infections and to coordinate innate and adaptive immunity $(2,13)$. Disturbances in macrophage function may lead to abnormal repair, such as uncontrolled production of inflammatory and growth mediators, deficient production of anti-inflammatory macrophages, or failed communication between macrophages and other non-immune cells, all of which are conducive to a status of persistent injury (18). Therefore, it is particularly important to elucidate the mechanism underlying macrophage activation. TLRs are membrane-associated PRRs that consist of an ectodomain with leucine-rich repeats (LRR) that mediate interactions with activator and coreceptors, a transmembrane region, and an intracellular TIR signaling domain (19). TLR3/4 are the main PRRs for recognition of Gram-negative bacterial LPS $(20,21)$ and viral dsRNA (22). Their binding to corresponding ligands leads to accumulation of intracellular TIR domains to enable recruitment of adapter proteins by TLR dimerization. The MyD88-dependent signaling pathway is activated by TLR4 from the cell surface (23), and then TLR4 translocates into the endosome in a GTPase- and CD14-dependent manner to trigger the TRIF-dependent signaling pathway that activates the 

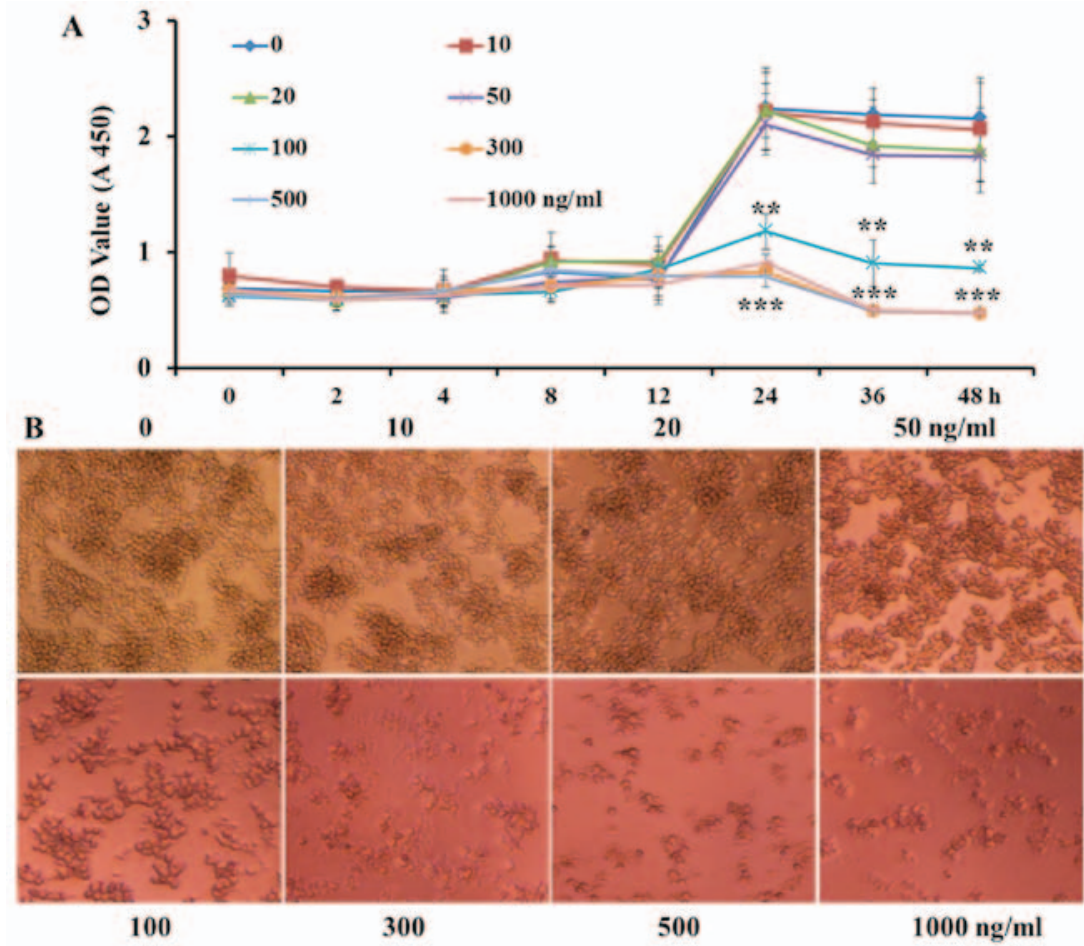

Figure 4. Vincristine (VCR) treatment at low concentration and for a short time did not inhibit cell proliferation. (A) The proliferation of RAW264.7 cells was assayed over a $48 \mathrm{~h}$ period with VCR of different concentration, and the data shown are means $\pm \mathrm{SD}$ from 3 independent experiments $\left({ }^{* * *} \mathrm{P}<0.01\right.$ and $\left.{ }^{* * * *} \mathrm{P}<0.001\right)$. (B) RAW264.7 cells were primed for $24 \mathrm{~h}$ with VCR at different concentrations, and cell morphology was observed under a light microscope (original magnification, x100). Similar observations were obtained from three independent experiments. OD, optical density.

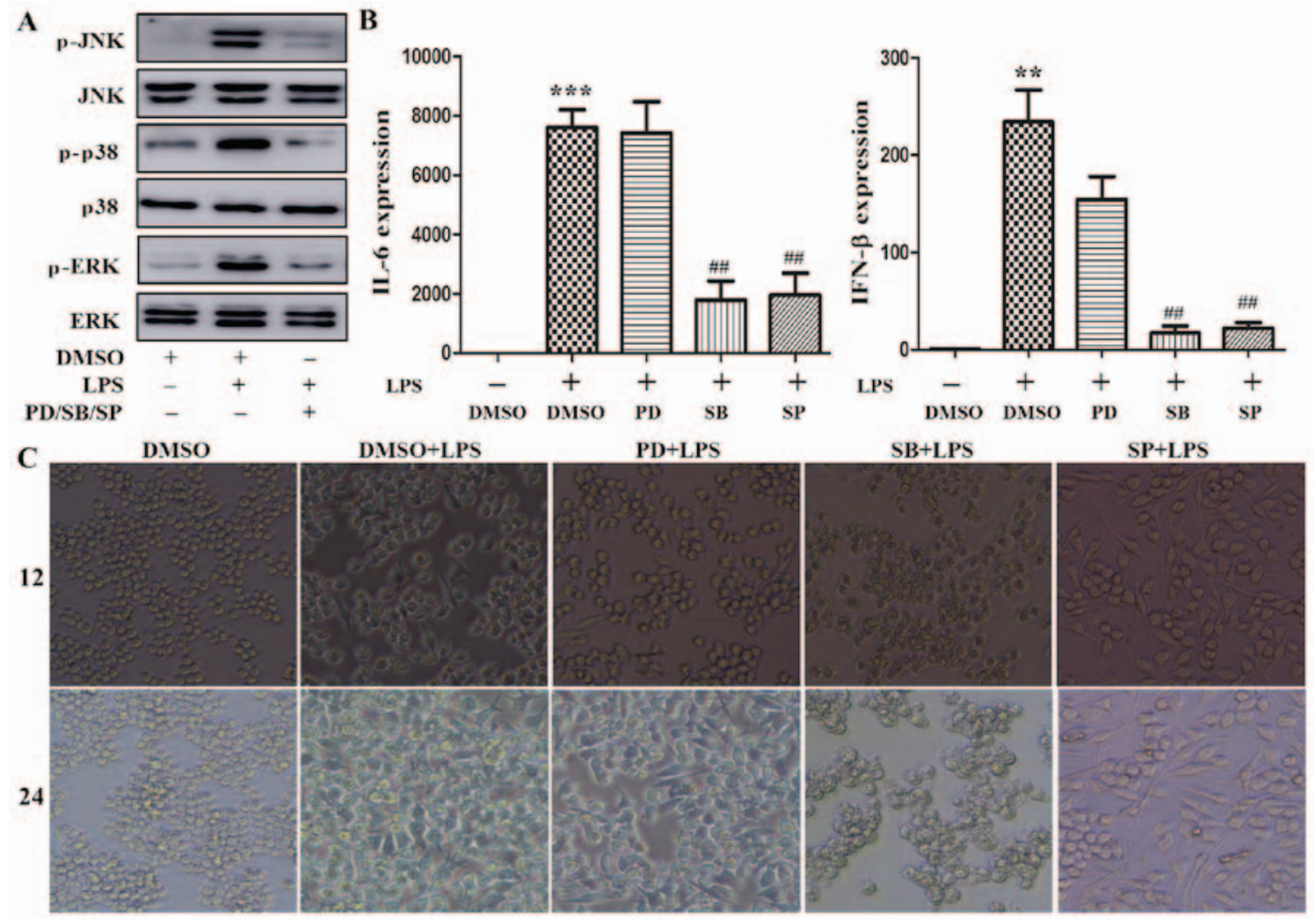

Figure 5. Mitogen-activated protein kinase (MAPK)/p38 signaling pathway inhibitor pretreatment repressed cytoskeleton rearrangement and production of interleukin (IL)-6 and interferon (IFN)- $\beta$. (A-C) RAW264.7 cells were primed for 30 min with PD98059, SB203580 and SP600125, and then stimulated with $100 \mathrm{ng} / \mathrm{ml}$ lipopolysaccharide (LPS). (A) The levels of non-phosphorylated and phosphorylated extracellular signal-regulated kinase (ERK), p38 and c-Jun $\mathrm{N}$-terminal kinase (JNK) in the lysates were detected by immunoblotting. (B) IL-6 and IFN- $\beta$ expression was measured by quantitative polymerase chain reaction $\left({ }^{* *} \mathrm{P}<0.05,{ }^{* * *} \mathrm{P}<0.001\right.$ and $\left.{ }^{\# \#} \mathrm{P}<0.01\right)$. (C) Cell morphology was observed under a light microscope (original magnification, $\mathrm{x} 100$ ). $\mathrm{RAW} 264.7$ cells were primed for $30 \mathrm{~min}$ with vincristine (VCR) and stimulated with $100 \mathrm{ng} / \mathrm{ml}$ LPS for $30 \mathrm{~min}$. Subsequently, p38 and p65 phosphorylation were detected by immunoblotting (D). Similar observations were obtained from three independent experiments. DMSO, dimethyl sulfoxide. 
TBK1-IRF3 to induce production of type $\approx$ I IFNs. TLR3 only utilizes the TRIF-dependent signaling pathway (19). The TLR signaling pathway is regulated at multiple levels, such as the expression of TLR (24), TLR signal complex assembly, ubiquitination and phosphorylation of associated proteins $(25,26)$, induction of negative and positive regulators $(13,26,27)$, and epigenetic and post-transcriptional modification regulation $(28,29)$. It was observed that the expression and secretion of IL-6, TNF- $\alpha$ and IL-12 p40 and p70 were always lower during poly(I:C) compared with LPS stimulation in previous studies $(16,30,31)$. Consistently, in the present study, it was demonstrated that IL- 6 and IFN- $\beta$ expression following poly(I:C) stimulation were always lower compared with LPS stimulation at any given timepoint, although the reason for this difference remains unclear.

The cytoskeleton plays a central role in cell morphology maintenance, cell migration and division, and organelle movement and localization; in addition, it is involved in intra- and extracellular signal transduction. The three elementary structural components of the cytoskeleton are microtubules, microfilaments and intermediate filaments via the polymerization and assembling of different monomers. These polymers undergo continual turnover and rearrangement and specifically bind different proteins to yield their respective functions. In the present study, a significant difference in cell morphology was observed between LPS and poly(I:C)-induced macrophage activation. LPS stimulation, but not poly(I:C) stimulation, significantly decreased tubulin protein expression, leading to cytoskeleton rearrangement. VCR, a drug accelerating microtubule depolymerization by binding to tubulin, inhibits cytoskeleton rearrangement in LPS-induced macrophages, decreasing the production of proinflammatory cytokines and diminishing cell migration. Thus, it was validated that cytoskeleton regulated LPS- but not poly(I:C)-induced macrophage activation. In addition, actin and GAPDH were found to be more stable as internal reference compared with tubulin in detecting the change in the expression of other proteins during LPS-induced macrophage activation.

The MAPK intracellular signaling pathway is a key mediator of TLR3/4-induced signal transduction. MAPKs, including ERK, p38 and JNK, regulate the synthesis of inflammatory mediators at the transcriptional and translational levels through $\mathrm{NF}-\kappa \mathrm{B}$ activation $(32,33)$. In the present study, it was demonstrated that pretreatment with PD98059, an EKR inhibitor, did not affect cytokine expression mediated by the MAPK/ERK signaling pathway. Similarly, it was observed that SB203580, a p38 signaling pathway inhibitor, repressed cytoskeleton rearrangement in LPS-induced macrophages and led to inhibition of the production of proinflammatory cytokines. PD98059 and SP600125, MAPK/ERK and JNK signaling pathway inhibitors, respectively, exerted no effect on cytoskeleton rearrangement leading to cytokine production. In previous studies, the MAPK signaling pathway was found to participate in tubulin and/or actin polymerization, regulating migration of vascular smooth muscle cells (34), podocyte response to ox-LDL (35) and hepatocellular cholestasis induced by oxidative stress (36). In addition, it was demonstrated that p38 phosphorylation, but not p65 phosphorylation, was ablated following VCR priming and LPS stimulation compared with LPS alone. In conclusion, our results demonstrated that the cytoskeleton played a different role in LPS- and poly(I:C)- induced macrophage activation. The MAPK/p38 signaling pathway, but not ERK and JNK, promoted cytoskeleton rearrangement in LPS-induced macrophage to promote the production of proinflammatory cytokines and cell migration. Given the pathological role of the macrophage inflammatory response in certain autoimmune diseases, VCR at low doses may be of therapeutic value in the treatment of autoimmune diseases with uncontrolled inflammatory response. In addition, due to the differences in the stability of cytoskeletal proteins, particularly tubulin, in LPS- and poly(I:C)-induced macrophage activation, GAPDH was used as an internal control to determine the expression level of the other proteins.

\section{Acknowledgements}

The present study was supported in part by grants from the National Natural Science Foundation of China (nos. 81472685 and81600469),the MajorSpecialPlanofScience and Technology of Shandong Province (no. 2015ZDXX0802A01), the Promotive Research Fund for Excellent Young and Middle-aged Scientisits of Shandong Province (no. BS2014YY037), and the Science and Technology Development Projects of Shandong Province (no. 2016GSF201126).

\section{References}

1. Pelka K, Shibata T, Miyake K and Latz E: Nucleic acid-sensing TLRs and autoimmunity: Novel insights from structural and cell biology. Immunol Rev 269: 60-75, 2016.

2. Kawai T and Akira S: The role of pattern-recognition receptors in innate immunity: Update on Toll-like receptors. Nat Immunol 11: 373-384, 2010.

3. Zhao W, Qi J, Wang L, Zhang M, Wang P and Gao C: LY294002 inhibits TLR3/4-mediated IFN- $\beta$ production via inhibition of IRF3 activation with a PI3K-independent mechanism. FEBS Lett 586: 705-710, 2012

4. Bosshart $\mathrm{H}$ and Heinzelmann M: Targeting bacterial endotoxin: Two sides of a coin. Ann NY Acad Sci 1096: 1-17, 2007.

5. Kustermans G, Piette J and Legrand-Poels S: Actin-targeting natural compounds as tools to study the role of actin cytoskeleton in signal transduction. Biochem Pharmacol 76: 1310-1322, 2008.

6. Diesel B, Hoppstädter J, Hachenthal N, Zarbock R, Cavelius C, Wahl B, Thewes N, Jacobs K, Kraegeloh A and Kiemer AK: Activation of Racl GTPase by nanoparticulate structures in human macrophages. Eur J Pharm Biopharm 84: 315-324, 2013.

7. Song W, Liu C and Upadhyaya A: The pivotal position of the actin cytoskeleton in the initiation and regulation of B cell receptor activation. Biochim Biophys Acta 1838: 569-578, 2014.

8. Chen YR, Feng F, Wang L, Qu SY, Zhang ZQ, Liu L, Qin HY, Liang YM and Han H: Deletion of RBP-J in dendritic cells compromises TLR-mediated DC activation accompanied by abnormal cytoskeleton reorganization. Mol Biol Rep 40: 1531-1539, 2013.

9. Moudi M, Go R, Yien CY and Nazre M: Vinca alkaloids. Int J Prev Med 4: 1231-1235, 2013.

10. Pathak P, Hess R and Weiss MA: Liposomal vincristine for relapsed or refractory $\mathrm{Ph}$-negative acute lymphoblastic leukemia: A review of literature. Ther Adv Hematol 5: 18-24, 2014.

11. Thakur V, Kush P, Pandey RS, Jain UK, Chandra R and Madan J: Vincristine sulfate loaded dextran microspheres amalgamated with thermosensitive gel offered sustained release and enhanced cytotoxicity in THP-1, human leukemia cells: In vitro and in vivo study. Mater Sci Eng C 61: 113-122, 2016.

12. Ruiz-Gómez MJ, Souviron A, Martínez-Morillo M and Gil L: P-glycoprotein, glutathione and glutathione S-transferase increase in a colon carcinoma cell line by colchicine. J Physiol Biochem 56: 307-312, 2000.

13. Qi J, Qiao Y, Wang P, Li S, Zhao W and Gao C: microRNA-210 negatively regulates LPS-induced production of proinflammatory cytokines by targeting NF- $\kappa \mathrm{B} 1$ in murine macrophages. FEBS Lett 586: 1201-1207, 2012 . 
14. Qi J,Li T, Bian H, Li F, Ju Y, Gao S, Su J, Ren W and Qin C: SNAI1 promotes the development of HCC through the enhancement of proliferation and inhibition of apoptosis. FEBS Open Bio 6: 326-337, 2016

15. Wu J, Zhang H, Hu B, Yang L, Wang P, Wang F and Meng X: Coptisine from Coptis chinensis inhibits production of inflammatory mediators in lipopolysaccharide-stimulated RAW264.7 murine macrophage cells. Eur J Pharmacol 780: 106-114, 2016.

16. Bode KA, Schmitz F, Vargas L, Heeg K and Dalpke AH: Kinetic of RelA activation controls magnitude of TLR-mediated IL-12p40 induction. J Immunol 182: 2176-2184, 2009.

17. Devisscher L, Verhelst X, Colle I, Van Vlierberghe H and Geerts A: The role of macrophages in obesity-driven chronic liver disease. J Leukoc Biol 99: 693-698, 2016.

18. Wynn TA and Vannella KM: Macrophages in tissue repair, regeneration, and fibrosis. Immunity 44: 450-462, 2016.

19. Beutler B: Microbe sensing, positive feedback loops, and the pathogenesis of inflammatory diseases. Immunol Rev 227 248-263, 2009

20. Alexopoulou L, Holt AC, Medzhitov R and Flavell RA: Recognition of double-stranded RNA and activation of NF-kappaB by Toll-like receptor 3. Nature 413: 732-738, 2001

21. Ruiz J, Kanagavelu S, Flores C, Romero L, Riveron R, Shih DQ and Fukata M: Systemic activation of TLR3-dependent TRIF signaling confers host defense against gram-negative bacteria in the intestine. Front Cell Infect Microbiol 5: 105, 2016.

22. Poltorak A, Smirnova I, Clisch R and Beutler B: Limits of a deletion spanning Tlr4 in $\mathrm{C} 57 \mathrm{BL} / 10 \mathrm{ScCr}$ mice. J Endotoxin Res 6: 51-56, 2000.

23. Kagan JC and Medzhitov R: Phosphoinositide-mediated adaptor recruitment controls Toll-like receptor signaling. Cell 125: 943-955, 2006.

24. Romieu-Mourez R, François M, Boivin MN, Bouchentouf M, Spaner DE and Galipeau J: Cytokine modulation of TLR expression and activation in mesenchymal stromal cells leads to a proinflammatory phenotype. J Immunol 182: 7963-7973, 2009.

25. Xiong $\mathrm{Y}$ and Medvedev AE: Induction of endotoxin tolerance in vivo inhibits activation of IRAK4 and increases negative regulators IRAK-M, SHIP-1, and A20. J Leukoc Biol 90: 1141-1148, 2011.

26. Xiong Y, Qiu F, Piao W, Song C, Wahl LM and Medvedev AE: Endotoxin tolerance impairs IL-1 receptor-associated kinase (IRAK) 4 and TGF-beta-activated kinase 1 activation, K63-linked polyubiquitination and assembly of IRAK1, TNF receptor-associated factor 6 , and IkappaB kinase gamma and increases A20 expression. J Biol Chem 286: 7905-7916, 2011.
27. Murphy MB, Xiong Y, Pattabiraman G, Manavalan TT, Qiu F and Medvedev AE: Pellino-3 promotes endotoxin tolerance and acts as a negative regulator of TLR2 and TLR4 signaling. J Leukoc Biol 98: 963-974, 2015.

28. Foster SL, Hargreaves DC and Medzhitov R: Gene-specific control of inflammation by TLR-induced chromatin modifications. Nature 447: 972-978, 2007.

29. O'Neill LA, Sheedy FJ and McCoy CE: MicroRNAs: The finetuners of Toll-like receptor signalling. Nat Rev Immunol 11: 163-175, 2011.

30. Zhao W, Wang L, Zhang M, Wang P, Qi J, Zhang L and Gao C: Nuclear to cytoplasmic translocation of heterogeneous nuclear ribonucleoprotein U enhances TLR-induced proinflammatory cytokine production by stabilizing mRNAs in macrophages. J Immunol 188: 3179-3187, 2012.

31. Zhang M, Wang L, Zhao X, Zhao K, Meng H, Zhao W and Gao C: TRAF-interacting protein (TRIP) negatively regulates IFN- $\beta$ production and antiviral response by promoting proteasomal degradation of TANK-binding kinase 1. J Exp Med 209: 1703-1711, 2012.

32. Chan ED and Riches DW: IFN-gamma + LPS induction of iNOS is modulated by ERK, JNK/SAPK, and p38(mapk) in a mouse macrophage cell line. Am J Physiol Cell Physiol 280: C441-C450, 2001.

33. Kaminska B: MAPK signalling pathways as molecular targets for anti-inflammatory therapy--from molecular mechanisms to therapeutic benefits. Biochim Biophys Acta 1754: 253-262, 2005.

34. Lee GL, Wu JY, Tsai CS, Lin CY, Tsai YT, Lin CS, Wang YF, Yet SF, Hsu YJ and Kuo CC: TLR4-activated MAPK-IL-6 axis regulates vascular smooth muscle cell function. Int J Mol Sci 17: E1394, 2016

35. Hu M, Fan M, Zhen J, Lin J, Wang Q, Lv Z and Wang R: FAK contributes to proteinuria in hypercholesterolaemic rats and modulates podocyte $\mathrm{F}$-actin re-organization via activating p38 in response to ox-LDL. J Cell Mol Med 21: 552-567, 2017.

36. Toledo FD, Basiglio CL, Barosso IR, Boaglio AC, Zucchetti AE, Sánchez Pozzi EJ and Roma MG: Mitogen-activated protein kinases are involved in hepatocanalicular dysfunction and cholestasis induced by oxidative stress. Arch Toxicol 91: 2391-2403, 2017 